\title{
Plasma phospholipid and dietary $\alpha$-linolenic acid, mortality, CHD and stroke: the Cardiovascular Health Study
}

\author{
Amanda M. Fretts ${ }^{1 *}$, Dariush Mozaffarian ${ }^{2}$, David S. Siscovick ${ }^{1}$, Colleen Sitlani ${ }^{1}$, Bruce M. Psaty ${ }^{1}$, \\ Eric B. Rimm ${ }^{2}$, Xiaoling Song ${ }^{3}$, Barbara McKnight ${ }^{1}$, Donna Spiegelman ${ }^{2}$, Irena B. King ${ }^{4}$ and \\ Rozenn N. Lemaitre ${ }^{1}$ \\ ${ }^{1}$ University of Washington, 1730 Minor Avenue, Suite 1360, Seattle, WA 98101, USA \\ ${ }^{2}$ Harvard University, 677 Huntington Avenue, Boston, MA 02115, USA \\ ${ }^{3}$ Fred Hutchinson Cancer Research Center, 1100 Fairview Avenue North, Seattle, WA 98109, USA \\ ${ }^{4}$ University of New Mexico, 1201 Camino de Salud North East, 1 UNM, Albuquerque, NM 87106, USA
}

(Submitted 10 October 2013 - Final revision received 29 April 2014 - Accepted 20 May 2014 - First published online 27 August 2014 )

\begin{abstract}
Previous studies have suggested that long-chain $n$-3 fatty acids derived from seafood are associated with a lower risk of mortality, CHD and stroke. Whether $\alpha$-linolenic acid (ALA, 18:3n-3), a plant-derived long-chain essential $n$ - 3 fatty acid, is associated with a lower risk of these outcomes is unclear. The aim of the present study was to examine the associations of plasma phospholipid and dietary ALA with the risk of mortality, CHD and stroke among older adults who participated in the Cardiovascular Health Study, a cohort study of adults aged $\geq 65$ years. A total of 2709 participants were included in the plasma phospholipid ALA analysis and 2583 participants were included in the dietary ALA analysis. Cox regression was used to assess the associations of plasma phospholipid and dietary ALA with the risk of mortality, incident CHD and stroke. In minimally and multivariable-adjusted models, plasma phospholipid ALA was found to be not associated with the risk of mortality, incident CHD or stroke. After adjustment for age, sex, race, enrolment site, education, smoking status, diabetes, BMI, alcohol consumption, treated hypertension and total energy intake, higher dietary ALA intake was found to be associated with a lower risk of total and non-cardiovascular mortality; on comparing the highest quintiles of dietary ALA with the lowest quintiles, the HR for total mortality and non-cardiovascular mortality were found to be 0.73 (95\% CI 0.61, 0.88) and 0.64 (95\% CI 0.52, 0.80), respectively. Dietary ALA was found to be not associated with the risk of cardiovascular mortality, incident CHD or stroke. In conclusion, the results of the present suggest study that dietary ALA, but not plasma phospholipid ALA, is associated with a lower risk of total and non-cardiovascular mortality in older adults.
\end{abstract}

Key words: Fatty acids: $\boldsymbol{\alpha}$-Linolenic acid: Mortality: CVD

Previous studies ${ }^{(1-7)}$ have suggested that long-chain $n$-3 fatty acids derived from seafood are associated with a lower risk of mortality, $\mathrm{CHD}$ and stroke. Whether $\alpha$-linolenic acid (ALA, 18:3n-3), a plant-derived long-chain essential $n$-3 fatty acid, is associated with a lower risk of these outcomes is less clear. Given concerns about the sustainability of fish populations and potential harm from fish contaminants ${ }^{(8,9)}$, a cheaper, plant-derived alternative source of $n-3$ fatty acids might be important to public health. Therefore, it is essential to understand whether plant-derived ALA exhibits similar associations with the risk of cardiovascular-related morbidity and mortality as seafood-derived $n-3$ fatty acids.
ALA is found in selected seed and vegetable oils, such as soyabean and rapeseed oils. These oils are used alone or in combination with other vegetable and seed oils in varying concentrations in many foods. Consequently, estimation of dietary ALA using FFQ is prone to measurement error. Plasma phospholipid ALA is an objective biomarker of circulating levels of ALA over the past 1-2 months that reflects diet together with the metabolism of dietary ALA. Thus, dietary and biomarker measures provide complementary information on ALA exposure. In the present study, we investigated the associations of both plasma phospholipid and dietary ALA with the risk of mortality, CHD and stroke among adults aged $\geq 65$ years who participated in the

Abbreviations: ALA, $\alpha$-linolenic acid; CHS, Cardiovascular Health Study; FADS2, $\Delta$-6-desaturase; LA, linoleic acid.

*Corresponding author: A. M. Fretts, fax +1 206287 2662, email amfretts@u.washington.edu 
Cardiovascular Health Study (CHS), a large community-based prospective cohort study.

\section{Experimental methods \\ Design and population}

The CHS is a community-based prospective cohort study of CVD and its risk factors among older adults from four geographical areas in the USA (Forsyth County, NC; Sacramento County, CA; Washington County, MD; and Allegheny County, PA). Previous publications have described the study rationale, study design and data collection methodology in detail $^{(10)}$. Briefly, non-institutionalised adults aged $\geq 65$ years were randomly selected and enrolled in the study using Medicare eligibility lists. In total, 5201 participants were enrolled in 1989-1990 and 687 participants (predominantly African Americans) were enrolled 3-4 years later. The study included annual clinic visits with interim phone calls from 1989 to 1999 and phone contact two times per year thereafter. The study was conducted according to the guidelines laid down in the Declaration of Helsinki, and all procedures involving human subjects were approved by the institutional review board of each centre. Written informed consent was obtained from all subjects/patients.

Among the 5565 study participants alive in 1992-3, fatty acids were quantified in 3941. After the exclusion of 1232 participants with prevalent CVD (myocardial infarction, angina, coronary revascularisation, stroke, transient ischaemic attack or heart failure) at the time of the 1992-3 blood draw, 2709 persons were included in the plasma phospholipid ALA analysis. Among the 3764 participants who completed a semiquantitative FFQ in 1996, 1181 had prevalent CVD at the time of questionnaire administration and were excluded from the analysis. The remaining 2583 participants comprised the analytical cohort for the dietary ALA analysis. In total, $80 \cdot 1 \%$ of the study participants included in the plasma phospholipid ALA analysis were also included in the dietary ALA analysis.

\section{Data collection}

Standardised interviews, physical examinations, medical history review, laboratory evaluations and diagnostic testing were performed at the annual clinic examinations. Fasting blood samples collected from all study participants were stored at $-70^{\circ} \mathrm{C}$.

\section{Plasma phospholipid $\alpha$-linolenic acid measurement}

Plasma phospholipid ALA was measured using blood samples collected from 1992 to 1993. Plasma lipids were extracted using the methods of Folch et al. ${ }^{(11)}$, as described previously. A one-dimensional TLC was used to separate phospholipids from neutral lipids. Phospholipid fractions were directly transesterified using the Lepage \& Roy ${ }^{(12)}$ method to prepare fatty acid methyl esters. Individual fatty acid methyl esters were separated using GC, as described previously (Agilent 5890 Gas Chromatograph flame ionisation detector; Agilent
Technologies; fused silica capillary column SP-2560 $(100 \mathrm{~m} \times 0.25 \mathrm{~mm}, 0.2 \mu \mathrm{m})$, Supelco Bellefonte; initial $160^{\circ} \mathrm{C}$ for $16 \mathrm{~min}$, ramp $3^{\circ} \mathrm{C} / \mathrm{min}$ to $240^{\circ} \mathrm{C}$, hold at $160^{\circ} \mathrm{C}$ for $15 \mathrm{~min})^{(5)}$. Laboratory inter-assay $\mathrm{CV}$ was $3 \cdot 1 \%$ for $\mathrm{ALA}^{(13)}$. All fatty acids were processed at the Biomarker Laboratory of the Fred Hutchinson Cancer Research Center (Seattle, WA USA). ALA is expressed as a percentage of total plasma phospholipid fatty acids analysed.

\section{Dietary $\alpha$-linolenic acid measurement}

Previous year's dietary intake was measured using a Willett 131-item semi-quantitative FFQ administered in 1996. This questionnaire has known reliability and validity ${ }^{(14)}$. To obtain a measure of dietary ALA, the dietary ALA content of each food item was multiplied with the participant's frequency response and then summed for all foods. For consistency with the measurement of plasma phospholipid ALA, which is assessed as a percentage of total fatty acids, dietary ALA was evaluated as a percentage of total fat, which is correlated better than absolute intake $(\mathrm{g} / \mathrm{d})$ or percentage of total energy with plasma phospholipid ALA $(r 0 \cdot 18)^{(15,16)}$.

\section{Mortality and CVD assessment}

Cause of death and cardiovascular events were adjudicated by a centralised CHS events committee based on information from medical records, laboratory/diagnostic reports, death certificates and/or interviews with next of kin. Details of CHS methods for surveillance and disease classification have been reported in detail previously ${ }^{(10,17)}$. For the purposes of this analysis, the association of plasma phospholipid or dietary ALA with the risk of total mortality, as well as CVD mortality, CHD mortality, and non-CVD mortality, and incident CHD and incident stroke was evaluated. Total CHD mortality was further subclassified as arrhythmic or non-arrhythmic deaths and strokes were subclassified as ischaemic, haemorrhagic or unknown type. The maximum duration of follow-up was 16 years for the plasma phospholipid ALA analysis and 12 years for the dietary ALA analysis.

\section{Statistical analyses}

Cox regression was used to examine the associations of plasma phospholipid and dietary ALA with the risk of total and causespecific mortality, incident CHD and incident stroke (total, ischaemic and haemorrhagic). Circulating ALA and dietary ALA were assessed both categorically (indicator quintiles) and semi-parametrically (cubic splines). Loss to follow-up was considered a censoring event. Schoenfeld's residuals were used to evaluate the proportional hazards assumption for both plasma phospholipid and dietary ALA. For consistency with the measurement of plasma phospholipid ALA (assessed as a percentage of total fatty acids), dietary ALA was adjusted for total energy to reduce measurement error and confounding by total reported energy ${ }^{(18)}$. Covariates of interest included age, sex, race (European American or African American), enrolment site (Bowman Grey, Davis, Hopkins or Pittsburgh), education 
(no high school, high school/vocational school, or college), smoking status (never, past or current), diabetes (yes/no), BMI $\left(\mathrm{kg} / \mathrm{m}^{2}\right)$, waist circumference $(\mathrm{cm})$, physical activity (kcal/week), alcohol consumption (drinks/week) and treated hypertension (yes/no).

In sensitivity analyses, further adjustment was made for linoleic acid (LA); LA is a major dietary PUFA that is present in many of the same foods containing ALA and may compete with ALA for elongation into longer-chain $n-3$ and $n-6$ fatty acids. Sensitivity analyses were also carried out by terminating follow-up at 8 years after the collection of plasma phospholipid or dietary ALA measures to minimise exposure misclassification, which may be higher in later years of follow-up.

The effects of potential interactions of ALA (modelled continuously) with sex, age, LA and $\Delta$-6-desaturase (FADS2) genotype on the risk of death or incident CHD or stroke was assessed. The statistical significance of each multiplicative interaction term was tested using likelihood ratio tests. Potential interactions of ALA and LA were examined as LA competes with ALA for elongation and desaturation into very-long-chain $n-3$ fatty acids $^{(19)}$. Similarly, genetic variability in FADS2 may affect the conversion of ALA to EPA and $\mathrm{DHA}^{(20)}$, so the interaction between FADS2 genotype and ALA was also examined. As dietary EPA and DHA may influence the associations of ALA with the outcomes of interest $^{(21)}$, stratification was done at the 25 th percentile of fish intake ( 0.6 servings $/ \mathrm{d})$ in sensitivity analyses.

Single imputation was used to impute missing values for covariates $(<2 \%$ missing values for all covariates) using data on age, sex, smoking status, education, race, BMI, physical activity, self-reported health status and diabetes at the time of ALA measurement. All statistical analyses were conducted using Stata version 10.0 (Stata Corporation).

\section{Results}

Association between plasma phospholipid $\alpha$-linolenic acid and risk of mortality, CHD and stroke

There were 2709 CHS participants free of CVD and with available plasma phospholipid ALA measures in 1992-3. Of these participants, 36.1\% were male, $90 \cdot 0 \%$ were Caucasian, and the median age was 73.0 years (interquartile range: $71 \cdot 0-98.0$ years). The baseline characteristics of the study participants according to quintile of plasma phospholipid ALA are given in online supplementary Table S1. There were 1757 deaths during 32111 person-years of follow-up. In both ageand sex- and fully adjusted analyses, plasma phospholipid ALA was not associated with the risk of total or cause-specific mortality (Table 1). Similarly, plasma phospholipid ALA was not associated with the risk of incident $\mathrm{CHD}$, stroke or

Table 1. Hazard ratios (HR) for the association of plasma phospholipid $\alpha$-linolenic acid with the risk of total and cause-specific mortality among 2709 US adults

(Hazard ratios and $95 \%$ confidence intervals)

\begin{tabular}{|c|c|c|c|c|c|c|c|c|c|c|}
\hline & \multicolumn{9}{|c|}{ Quintiles } & \multirow[b]{3}{*}{$P$ for treno } \\
\hline & \multirow[b]{2}{*}{ I } & \multicolumn{2}{|r|}{ II } & \multicolumn{2}{|r|}{ III } & \multicolumn{2}{|r|}{ IV } & \multicolumn{2}{|r|}{ V } & \\
\hline & & HR & $95 \% \mathrm{Cl}$ & HR & $95 \% \mathrm{Cl}$ & $\mathrm{HR}$ & $95 \% \mathrm{Cl}$ & $\mathrm{HR}$ & $95 \% \mathrm{Cl}$ & \\
\hline \multicolumn{11}{|l|}{ Total mortality } \\
\hline Person-years & 6483 & \multicolumn{2}{|r|}{6025} & \multicolumn{2}{|r|}{6315} & \multicolumn{2}{|r|}{6352} & \multicolumn{2}{|r|}{6936} & \\
\hline No. of deaths & 360 & \multicolumn{2}{|r|}{354} & \multicolumn{2}{|r|}{359} & & 331 & \multicolumn{2}{|r|}{353} & \\
\hline Age- and sex-adjusted & 1.0 (ref.) & 1.08 & $0.93,1.25$ & 1.07 & $0.92,1.23$ & 0.94 & $0.81,1.10$ & 0.91 & $0.79,1.06$ & 0.06 \\
\hline Additionally adjusted model ${ }^{*}$ & 1.0 (ref.) & 1.09 & $0.93,1.26$ & 1.09 & $0.94,1.27$ & 0.95 & $0.81,1 \cdot 11$ & 0.93 & $0.79,1.08$ & 0.11 \\
\hline \multicolumn{11}{|l|}{ Non-CVD mortality } \\
\hline No. of deaths & 235 & \multicolumn{2}{|r|}{229} & \multicolumn{2}{|r|}{236} & \multicolumn{2}{|r|}{209} & \multicolumn{2}{|r|}{229} & \\
\hline Age- and sex-adjusted & 1.0 (ref.) & 1.07 & $0.89,1.28$ & 1.08 & $0.90,1.29$ & 0.92 & $0.76,1 \cdot 11$ & 0.91 & $0.76,1.09$ & $0 \cdot 10$ \\
\hline Additionally adjusted model ${ }^{*}$ & 1.0 (ref.) & 1.09 & $0.90,1.31$ & $1 \cdot 11$ & $0.92,1.34$ & 0.92 & $0.76,1 \cdot 11$ & 0.90 & $0.75,1.09$ & 0.09 \\
\hline \multicolumn{11}{|l|}{ Total CVD mortality } \\
\hline No. of deaths & 101 & \multicolumn{2}{|r|}{108} & \multicolumn{2}{|r|}{102} & \multicolumn{2}{|r|}{102} & \multicolumn{2}{|r|}{106} & \\
\hline Age- and sex-adjusted & 1.0 (ref.) & $1 \cdot 16$ & $0.88,1.52$ & 1.07 & $0.81,1.41$ & 1.02 & $0.78,1.35$ & 0.96 & $0.73,1.27$ & 0.50 \\
\hline Additionally adjusted model ${ }^{*}$ & 1.0 (ref.) & $1 \cdot 15$ & $0.87,1.53$ & 1.08 & $0.81,1.44$ & 1.05 & $0.79,1.40$ & 1.02 & $0.77,1.36$ & 0.87 \\
\hline \multicolumn{11}{|l|}{ Total CHD mortality } \\
\hline No. of deaths & 68 & \multicolumn{2}{|r|}{64} & \multicolumn{2}{|r|}{69} & \multicolumn{2}{|r|}{64} & \multicolumn{2}{|r|}{66} & \\
\hline Age- and sex-adjusted & 1.0 (ref.) & 1.03 & $0.74,1.46$ & $1 \cdot 11$ & $0.79,1.56$ & 0.99 & $0.70,1.40$ & 0.92 & $0.66,1.30$ & 0.59 \\
\hline Additionally adjusted model ${ }^{*}$ & 1.0 (ref.) & 1.06 & $0.75,1.51$ & $1 \cdot 16$ & $0.82,1.65$ & 1.02 & $0.71,1.45$ & 1.03 & $0.72,1.46$ & 0.98 \\
\hline Arrhythmic death & & & & & & & & & & \\
\hline No. of deaths & 35 & & 30 & & 38 & & 34 & & 33 & \\
\hline Age- and sex-adjusted & 1.0 (ref.) & 0.93 & $0.57,1.52$ & $1 \cdot 16$ & $0.73,1.83$ & 1.00 & $0.62,1.60$ & 0.88 & $0.55,1.42$ & 0.72 \\
\hline Additionally adjusted model ${ }^{*}$ & 1.0 (ref.) & 1.02 & $0.62,1.67$ & $1 \cdot 23$ & $0.76,1.99$ & 1.05 & $0.64,1.71$ & 0.98 & $0.60,1.62$ & 0.99 \\
\hline Non-arrhythmic death & & & & & & & & & & \\
\hline No. of deaths & 33 & & 34 & & 31 & & 30 & & 33 & \\
\hline Age- and sex-adjusted & 1.0 (ref.) & $1 \cdot 14$ & $0.71,1.85$ & 1.06 & $0.65,1.74$ & 0.98 & $0.60,1.62$ & 0.97 & $0.59,1.57$ & 0.70 \\
\hline Additionally adjusted model ${ }^{*}$ & 1.0 (ref.) & $1 \cdot 12$ & $0.68,1.83$ & 1.09 & $0.65,1.81$ & 0.99 & $0.59,1.66$ & 1.07 & $0.65,1.76$ & 0.97 \\
\hline
\end{tabular}

ref., Reference.

${ }^{*}$ Additionally adjusted for race, enrolment site, education, smoking status, diabetes, BMI, waist circumference, physical activity, alcohol consumption and treated hypertension. 
Table 2. Hazard ratios (HR) for the association of plasma phospholipid $\alpha$-linolenic acid with the risk of incident stroke and CHD among 2709 US adults (Hazard ratios and $95 \%$ confidence intervals)

\begin{tabular}{|c|c|c|c|c|c|c|c|c|c|c|}
\hline & \multicolumn{9}{|c|}{ Quintiles } & \multirow[b]{3}{*}{$P$ for trend } \\
\hline & \multirow[b]{2}{*}{1} & \multicolumn{2}{|r|}{ II } & \multicolumn{2}{|r|}{ III } & \multicolumn{2}{|r|}{ IV } & \multicolumn{2}{|r|}{ V } & \\
\hline & & $\mathrm{HR}$ & $95 \% \mathrm{Cl}$ & $\mathrm{HR}$ & $95 \% \mathrm{Cl}$ & $\mathrm{HR}$ & $95 \% \mathrm{Cl}$ & $\mathrm{HR}$ & $95 \% \mathrm{Cl}$ & \\
\hline \multicolumn{11}{|l|}{ Total stroke } \\
\hline Person-years & 6208 & & 5792 & & 6026 & & 6132 & & 6589 & \\
\hline No. of cases & 85 & & 80 & & 94 & & 80 & & 91 & \\
\hline Age- and sex-adjusted & 1.0 (ref.) & 1.00 & $0.74,1.36$ & 1.11 & $0.83,1.50$ & 0.91 & $0.67,1.24$ & 0.98 & $0.73,1.32$ & 0.68 \\
\hline Additionally adjusted model ${ }^{\star}$ & 1.0 (ref.) & 0.96 & $0 \cdot 70,1 \cdot 31$ & $1 \cdot 10$ & $0.81,1.49$ & 0.88 & $0.64,1 \cdot 20$ & 0.97 & $0.71,1.31$ & 0.66 \\
\hline \multicolumn{11}{|l|}{ Ischaemic stroke } \\
\hline No. of cases & 69 & & 63 & & 70 & & 62 & & 73 & \\
\hline Age- and sex-adjusted & 1.0 (ref.) & 0.97 & $0.69,1.37$ & 1.03 & $0.74,1.43$ & 0.88 & $0.62,1.24$ & 0.97 & $0.70,1.35$ & 0.69 \\
\hline Additionally adjusted model ${ }^{*}$ & 1.0 (ref.) & 0.92 & $0.65,1.30$ & 1.01 & $0.72,1.43$ & 0.84 & $0.59,1.20$ & 0.97 & $0.69,1.36$ & 0.72 \\
\hline \multicolumn{11}{|l|}{ Haemorrhagic stroke } \\
\hline No. of cases & 11 & & 10 & & 15 & & 11 & & 12 & \\
\hline Age- and sex-adjusted & 1.0 (ref.) & 0.97 & $0.41,2.28$ & 1.38 & $0.63,3.02$ & 0.96 & $0.41,2.22$ & 0.99 & $0.44,2.26$ & 0.96 \\
\hline Additionally adjusted model ${ }^{*}$ & 1.0 (ref.) & 1.01 & $0.42,2.43$ & 1.45 & $0.65,3.27$ & 0.94 & $0.39,2.26$ & 0.95 & $0.40,2.25$ & 0.83 \\
\hline \multicolumn{11}{|l|}{ Total CHD } \\
\hline Person-years & 6124 & & 5756 & & 5995 & & 5928 & & 6406 & \\
\hline No. of cases & 83 & & 80 & & 81 & & 92 & & 90 & \\
\hline Age- and sex-adjusted & 1.0 (ref.) & 1.08 & $0.79,1.47$ & 1.09 & $0.80,1.49$ & 1.25 & $0.93,1.68$ & 1.14 & $0.85,1.54$ & 0.23 \\
\hline Additionally adjusted model ${ }^{\star}$ & 1.0 (ref.) & $1 \cdot 10$ & $0.80,1.50$ & 1.10 & $0.80,1.52$ & 1.21 & $0.88,1.64$ & 1.22 & $0.90,1.68$ & 0.16 \\
\hline
\end{tabular}

ref., Reference.

*Additionally adjusted for race, enrolment site, education, smoking status, diabetes, BMI, waist circumference, physical activity, alcohol consumption and treated hypertension.

stroke subtypes (Table 2). Using restricted cubic splines to model plasma phospholipid ALA, additionally adjusting for plasma phospholipid LA or fish intake, terminating followup at 8 years, or performing subgroup analyses by sex or fish intake had no meaningful effect on reported hazard ratios (data not shown). There was no evidence of interactions of ALA with age, sex, FADS2 genotype or plasma phospholipid LA on the risk of death, CHD or stroke (data not shown).

\section{Association between dietary $\alpha$-linolenic acid and risk of mortality, CHD and stroke}

In general, the characteristics of the study participants according to quintile of dietary ALA were similar to those according to quintile of plasma phospholipid ALA, except for the absence of observed differences in lipid-lowering medication use or BMI according to quintile of dietary ALA (online supplementary Table S2). In total, 1517 deaths occurred during 25849 personyears of follow-up among participants with dietary intake data. In both minimally and fully adjusted analyses, higher dietary ALA intake was found to be associated with a lower risk of total mortality and non-CVD mortality (Table 3). Hazard ratios did not change materially after additionally adjusting for other dietary factors, such as dietary LA or fish intake. There were no statistically significant associations of dietary ALA with the risk of total CVD mortality, total CHD mortality or CHD-related non-arrhythmic or arrhythmic deaths after adjustment for age, sex, energy intake, race, enrolment site, education, smoking status, alcohol consumption, BMI, diabetes and treated hypertension (Table 3). There was no significant association of dietary ALA with the risk of incident CHD or stroke (Table 4). Similar to the results of the plasma phospho- lipid analysis, it was found that additionally adjusting for dietary LA or fish intake, terminating follow-up at 8 years, or performing subgroup analyses by fish intake did not affect the results (data not shown). There was also no evidence of interactions between dietary ALA and age, sex, FADS2 genotype or LA on the risk of death, CHD or stroke (data not shown).

In exploratory secondary analyses, the association of dietary ALA with the risk of non-CVD mortality subtypes (i.e. deaths from dementia, cancer, infection, trauma/fracture and respiratory diseases) was further examined. Approximately $60 \%$ of the participants who died from non-CVD causes had cancer or dementia listed as causes of death in death records. Higher dietary ALA intake was associated with a lower risk of death from dementia and cancer. There were no statistically significant associations of dietary ALA with the risk of deaths from respiratory diseases, infection or trauma/fracture, although power was limited due to a small number of deaths from these causes (online supplementary Table S3).

\section{Discussion}

In this large prospective cohort study of older adults, we found no significant associations of plasma phospholipid ALA with the risk of total or cause-specific mortality, CHD or stroke. Dietary ALA was associated with a lower risk of total mortality, which appeared to be related to a significantly lower risk of non-CVD deaths. Dietary ALA was not associated with the risk of CVD mortality, CHD or stroke.

It is interesting that higher dietary ALA intake was associated with a lower risk of total mortality in the study cohort. When specific types of deaths were evaluated, this inverse association was only statistically significant for non-CVD 
Table 3. Hazard ratios (HR) for the association of dietary $\alpha$-linolenic acid with the risk of total and cause-specific mortality among 2583 US adults (Hazard ratios and $95 \%$ confidence intervals)

\begin{tabular}{|c|c|c|c|c|c|c|c|c|c|c|}
\hline & \multicolumn{9}{|c|}{ Quintiles } & \multirow[b]{3}{*}{$P$ for trend } \\
\hline & \multirow[b]{2}{*}{1} & \multicolumn{2}{|r|}{ II } & \multicolumn{2}{|r|}{ III } & \multicolumn{2}{|r|}{ IV } & \multicolumn{2}{|r|}{$\mathrm{V}$} & \\
\hline & & $\mathrm{HR}$ & $95 \% \mathrm{Cl}$ & $\mathrm{HR}$ & $95 \% \mathrm{Cl}$ & $\mathrm{HR}$ & $95 \% \mathrm{Cl}$ & HR & $95 \% \mathrm{Cl}$ & \\
\hline \multicolumn{11}{|l|}{ Total mortality } \\
\hline Person-years & 4875 & \multicolumn{2}{|r|}{4987} & \multicolumn{2}{|r|}{5096} & \multicolumn{2}{|r|}{5291} & \multicolumn{2}{|r|}{5600} & \\
\hline No. of deaths & 328 & \multicolumn{2}{|r|}{328} & \multicolumn{2}{|r|}{301} & \multicolumn{2}{|r|}{298} & \multicolumn{2}{|r|}{262} & \\
\hline Age-sex- and energy-adjusted & 1.0 (ref.) & 0.93 & $0.80,1.09$ & 0.85 & $0.73,0.99$ & 0.83 & $0.71,0.97$ & 0.70 & $0.59,0.82$ & $<0.0001$ \\
\hline Additionally adjusted model ${ }^{\star}$ & 1.0 (ref.) & 0.98 & $0.84,1 \cdot 15$ & 0.88 & $0.75,1.03$ & 0.86 & $0.73,1.02$ & 0.73 & $0.61,0.88$ & $<0.0001$ \\
\hline \multicolumn{11}{|l|}{ Non-CVD mortality } \\
\hline No. of deaths & 225 & \multicolumn{2}{|r|}{225} & \multicolumn{2}{|r|}{204} & \multicolumn{2}{|r|}{186} & \multicolumn{2}{|r|}{161} & \\
\hline Age-sex- and energy-adjusted & 1.0 (ref.) & 0.94 & $0.78,1.13$ & 0.84 & $0.69,1.02$ & 0.75 & $0.62,0.92$ & 0.62 & $0.51,0.77$ & $<0.0001$ \\
\hline Additionally adjusted model* & 1.0 (ref.) & 0.98 & $0.81,1.18$ & 0.87 & $0.71,1.05$ & 0.79 & $0.65,0.97$ & 0.64 & $0.52,0.80$ & $<0.0001$ \\
\hline \multicolumn{11}{|l|}{ Total CVD mortality } \\
\hline No. of deaths & 89 & & 83 & \multicolumn{2}{|r|}{76} & \multicolumn{2}{|r|}{92} & \multicolumn{2}{|r|}{89} & \\
\hline Age-sex- and energy-adjusted & 1.0 (ref.) & 0.86 & $0.64,1.17$ & 0.79 & $0.58,1.07$ & 0.94 & $0.70,1.26$ & 0.88 & $0.65,1.18$ & 0.61 \\
\hline Additionally adjusted model ${ }^{\star}$ & 1.0 (ref.) & 0.93 & $0.68,1.25$ & 0.83 & $0.61,1.14$ & 0.97 & $0.72,1.31$ & 0.96 & $0.71,1.32$ & 0.92 \\
\hline \multicolumn{11}{|l|}{ Total CHD mortality } \\
\hline No. of deaths & 61 & & 55 & \multicolumn{2}{|r|}{50} & \multicolumn{2}{|r|}{62} & \multicolumn{2}{|r|}{52} & \\
\hline Age-sex- and energy-adjusted & 1.0 (ref.) & 0.84 & $0.59,1.22$ & 0.77 & $0.53,1.11$ & 0.95 & $0.66,1.36$ & 0.77 & $0.53,1.13$ & 0.35 \\
\hline Additionally adjusted model ${ }^{*}$ & 1.0 (ref.) & 0.89 & $0.62,1.29$ & 0.83 & $0.57,1.21$ & 0.94 & $0.65,1.36$ & 0.85 & $0.58,1.26$ & 0.54 \\
\hline \multicolumn{11}{|l|}{ Arrhythmic death } \\
\hline No. of deaths & 30 & & 28 & & 23 & & 34 & & 20 & \\
\hline Age-sex- and energy-adjusted & 1.0 (ref.) & 0.87 & $0.52,1.45$ & 0.72 & $0.42,1.24$ & 1.05 & $0.64,1.73$ & 0.61 & $0.34,1.08$ & 0.26 \\
\hline Additionally adjusted model ${ }^{*}$ & 1.0 (ref.) & 0.93 & $0.55,1.58$ & 0.80 & $0.46,1.38$ & $1 \cdot 10$ & $0.66,1.84$ & 0.68 & $0.38,1.23$ & 0.42 \\
\hline Non-arrhythmic death & & & & & & & & & & \\
\hline No. of deaths & 31 & & 27 & & 27 & & 28 & & 32 & \\
\hline Age-sex- and energy-adjusted & 1.0 (ref.) & 0.82 & $0.49,1.38$ & 0.81 & $0.49,1.36$ & 0.85 & $0.50,1.42$ & 0.93 & $0.56,1.54$ & 0.85 \\
\hline Additionally adjusted model ${ }^{*}$ & 1.0 (ref.) & 0.86 & $0.51,1.44$ & 0.87 & $0.52,1.45$ & 0.79 & $0.46,1.36$ & 1.02 & $0.61,1.71$ & 0.94 \\
\hline
\end{tabular}

ref., Reference.

${ }^{*}$ Additionally adjusted for race, enrolment site, education, smoking status, diabetes, BMI, alcohol consumption and treated hypertension.

deaths, in particular, deaths due to cancer and dementia. Biological mechanisms by which dietary ALA may reduce the risk of non-CVD mortality are not well established. In vitro and rodent studies of cancer suggest that ALA may suppress cancer cell proliferation, inhibit tumour growth and increase apoptosis ${ }^{(22-24)}$. Additionally, animal models indicate that ALA deficiency may promote abnormalities in cerebral structures, and it is hypothesised that low levels of ALA may be associated with poor cognitive function in humans ${ }^{(25)}$. However, the results of epidemiological studies that have examined the relationships of ALA with cancer or dementia in human populations are conflicting ${ }^{(25-29)}$. More research is needed to better understand the mechanisms by which ALA may be associated with a lower risk of non-CVD mortality.

On the other hand, the inverse associations of dietary ALA with the risk of total mortality and non-CVD mortality might also be due to chance or to residual confounding by other poorly measured or unmeasured factors related to both dietary ALA and death risk. Our findings support the need for further investigation of the relationship of habitual dietary ALA consumption and total and cause-specific mortality risk.

Very few previous studies have evaluated the association of circulating ALA with the risk of incident CVD, stroke and mortality. The results of a small prospective nested case-control study among 192 men in the USA indicated that ALA in cholesterol esters is associated with a lower risk of stroke $\mathrm{e}^{(30)}$, while a retrospective case-control study among 134 South Koreans found no significant association of ALA in erythrocytes with stroke risk ${ }^{(31)}$. In four previous prospective studies that have examined the association of circulating ALA with the risk of incident CHD, stroke or mortality, circulating ALA was found to be not significantly associated with the development of $\mathrm{CHD}^{(32,33)}$, stroke ${ }^{(34)}$ or total/CVD mortality ${ }^{(35)}$. Our findings are also consistent with the results of a recent meta-analysis that found no statistically significant association of circulating ALA with the risk of fatal and non-fatal CHD or stroke ${ }^{(36)}$.

Relatively few studies have assessed the relationship of dietary ALA with the risk of mortality, stroke and CHD, and the findings are inconsistent. The results of the present study support the findings of the Nurses' Health Study that found dietary ALA to be associated with a lower risk of all-cause mortality ${ }^{(37)}$. They are also consistent with the findings of seven prospective studies ${ }^{(38-44)}$ and a small meta-analysis ${ }^{(45)}$ that indicate no association of ALA with the risk of non-fatal or fatal heart disease and with those of two prospective studies $^{(46,47)}$ that found no association of dietary ALA with the risk of stroke. Our findings are also consistent with the results of a recent meta-analysis of previous observational studies in which no association of dietary ALA with the risk of CHD or stroke was found ${ }^{(36)}$. On the other hand, the results of the meta-analysis showed a modest inverse association of dietary ALA with the risk of fatal CHD. The results of the present study are inconsistent with those of two studies in which higher dietary ALA intake was found to be associated with a lower risk of fatal $\mathrm{IHD}^{(48)}$ and sudden cardiac death ${ }^{(42)}$ among women who participated in the Nurses' Health 
Table 4. Hazard ratios (HR) for the association of dietary $\alpha$-linolenic acid with the risk of incident stroke and CHD among 2583 adults (Hazard ratios and $95 \%$ confidence intervals)

\begin{tabular}{|c|c|c|c|c|c|c|c|c|c|c|}
\hline & \multicolumn{9}{|c|}{ Quintiles } & \multirow[b]{3}{*}{$P$ for trend } \\
\hline & \multirow[b]{2}{*}{ I } & \multicolumn{2}{|r|}{ II } & \multicolumn{2}{|r|}{ III } & \multicolumn{2}{|r|}{ IV } & \multicolumn{2}{|r|}{ V } & \\
\hline & & $\mathrm{HR}$ & $95 \% \mathrm{Cl}$ & $\mathrm{HR}$ & $95 \% \mathrm{Cl}$ & $\mathrm{HR}$ & $95 \% \mathrm{Cl}$ & $\mathrm{HR}$ & $95 \% \mathrm{Cl}$ & \\
\hline \multicolumn{11}{|l|}{ Total stroke } \\
\hline Person-years & 4691 & & 4785 & & 4891 & & 4997 & & 5380 & \\
\hline No. of cases & 70 & & 64 & & 75 & & 81 & & 68 & \\
\hline Age-sex- and energy-adjusted & 1.0 (ref.) & 0.86 & $0.61,1 \cdot 21$ & 1.00 & $0.72,1.39$ & 1.05 & $0.76,1.45$ & 0.84 & $0.60,1.17$ & 0.69 \\
\hline Additionally adjusted model* & 1.0 (ref.) & 0.89 & $0.64,1.26$ & 0.97 & $0.70,1.35$ & 1.09 & $0.78,1.51$ & 0.86 & $0.60,1 \cdot 21$ & 0.80 \\
\hline \multicolumn{11}{|l|}{ Ischaemic stroke } \\
\hline No. of cases & 59 & & 52 & & 54 & & 67 & & 46 & \\
\hline Age-sex- and energy-adjusted & 1.0 (ref.) & 0.85 & $0.59,1.23$ & 0.85 & $0.59,1.24$ & 1.03 & $0.72,1.47$ & 0.67 & $0.45,1.01$ & 0.19 \\
\hline Additionally adjusted model ${ }^{*}$ & 1.0 (ref.) & 0.89 & $0.61,1.30$ & 0.84 & $0.58,1.22$ & 1.08 & $0.75,1.54$ & 0.70 & $0.47,1.04$ & 0.29 \\
\hline \multicolumn{11}{|l|}{ Haemorrhagic stroke } \\
\hline No. of cases & 8 & & 8 & & 15 & & 10 & & 15 & \\
\hline Age-sex-and energy-adjusted & 1.0 (ref.) & $1 \cdot 26$ & $0.44,3.63$ & 2.36 & $0.91,6.08$ & 1.57 & $0.57,4.36$ & $2 \cdot 29$ & $0.88,5.99$ & 0.09 \\
\hline Additionally adjusted model ${ }^{\star}$ & 1.0 (ref.) & 1.19 & $0.41,3.44$ & $2 \cdot 12$ & $0.81,5.54$ & 1.52 & $0.54,4.24$ & 1.96 & $0.73,5 \cdot 27$ & 0.16 \\
\hline \multicolumn{11}{|l|}{ Total CHD } \\
\hline Person-years & 4631 & & 4740 & & 4815 & & 4960 & & 5321 & \\
\hline No. of cases & 77 & & 71 & & 67 & & 92 & & 71 & \\
\hline Age-sex- and energy-adjusted & 1.0 (ref.) & 0.92 & $0.67,1.27$ & 0.87 & $0.63,1.21$ & 1.25 & $0.92,1.70$ & 0.92 & $0.66,1.28$ & 0.67 \\
\hline Additionally adjusted model ${ }^{*}$ & 1.0 (ref.) & 0.97 & $0.70,1.34$ & 0.88 & $0.63,1.23$ & 1.25 & $0.91,1.70$ & 0.93 & $0.67,1.30$ & 0.75 \\
\hline
\end{tabular}

ref., Reference.

${ }^{\star}$ Additionally adjusted for race, enrolment site, education, smoking status, diabetes, BMI, alcohol consumption and treated hypertension.

Study. Although our analyses did not specifically assess the association of dietary ALA with the risk of fatal IHD or sudden cardiac death, we found no statistically significant association of dietary ALA with the risk of cardiovascularrelated deaths. Our findings are also discordant with the findings of a large Dutch study in which dietary ALA was found to be inversely associated with the risk of stroke. In that study, participants in the upper four quintiles of dietary ALA had a 35-50\% lower risk of stroke when compared with those in the lowest quintile of dietary ALA ${ }^{(39)}$. Inconsistencies between the results of these studies may be due to underlying differences between the populations studied (e.g. differences in age, background diet or other health factors). For instance, participants of the Dutch study that reported an inverse association of dietary ALA with the risk of stroke were younger (mean 41.5 (SD 11.1) years at baseline) and had higher reported dietary ALA intake (mean dietary ALA intake for women and men: $1.2(\mathrm{SD} \quad 0.5)$ and $1.6(\mathrm{SD} \quad 0.6) \mathrm{g} / \mathrm{d}$, respectively) when compared with the participants of the CHS. Inconsistencies between the results of these studies may also be due to measurement error.

The present study has several strengths. The prospective analysis and cohort design reduced potential for both recall bias and selection bias. Both plasma phospholipid ALA and dietary ALA were assessed, providing complementary measures of exposure to this plant-derived $n-3$ fatty acid. Focus was on older adults - a population at a high risk of mortality, CHD and stroke. Detailed information on demographics, enrolment site and lifestyle habits was collected using standardised instruments, increasing our ability to adjust for confounding. The community-based enrolment of the cohort increased generalisability.
The present study also has several limitations. Plasma phospholipid ALA and dietary ALA were assessed once, and levels may have changed during the follow-up. Nevertheless, terminating follow-up at 8 years, which would minimise effects of misclassification, did not alter the results. For the purposes of the present study, dietary ALA and plasma phospholipid ALA were considered as complimentary measures of ALA exposure. However, plasma phospholipid ALA levels were low ( $<1 \%$ total fatty acids), and the correlation between plasma phospholipid ALA and dietary ALA was modest. It is possible that other tissue compartments with higher proportions of ALA (e.g. adipose tissue) may be a better marker of dietary ALA intake, and more studies are needed to examine the association of other biomarkers of ALA with the risk of mortality, CHD and stroke. Additional limitations include errors in dietary ALA measurement, which if random could attenuate findings towards the null. Although we included several major risk factors as covariates in our analyses of the association of ALA with the risk of mortality, CHD and stroke, residual confounding by unknown or poorly measured factors is possible. As ALA competes with LA for elongation/desaturation, the absolute intakes of ALA and LA are important determinants of ALA metabolism, and the association of ALA with the risk of mortality, CHD and stroke may differ in populations following other diets (e.g. populations with very low intakes of LA). Similarly, all study participants were aged $\geq 65$ years, and the generalisability of these findings to younger populations is not known. On the other hand, we found little evidence for effect modification in analyses stratified by LA intake or age.

In summary, in this large prospective cohort of older adults, neither plasma phospholipid nor dietary ALA was associated with CVD mortality, CHD or stroke. The inverse association 
of dietary ALA with the risk of total mortality and non-CVD mortality requires further investigation and replication in other studies.

\section{Supplementary material}

To view supplementary material for this article, please visit http://dx.doi.org/10.1017/S0007114514001925

\section{Acknowledgements}

The authors express their gratitude to the participants of the CHS.

The present study was financially supported by HL085710-01 from the National Heart, Lung, and Blood Institute (NHLBI), with co-funding from the Office of Dietary Supplements. It was also supported by HHSN268201200036C, HHSN26820080 0007C, N01HC55222, N01HC85079, N01HC85080, N01HC8 5081, N01HC85082, N01HC85083, N01HC85086, and grant HL080295 from the NHLBI, with additional contribution from the National Institute of Neurological Disorders and Stroke (NINDS). Additional support was provided by AG023629 from the National Institute on Aging (NIA). A full list of principal CHS investigators and institutions can be found at http://www.chsnhlbi.org/pi.htm. The NHLBI, Office of Dietary Supplements, NINDS and NIA had no role in the design and analysis of the study or in the writing of this article. The content is solely the responsibility of the authors and does not necessarily represent the official views of the National Institutes of Health.

The authors' contributions are as follows: A. M. F. performed the literature review and data analysis as well as wrote the manuscript; R. N. L., D. M. and D. S. S. were the senior investigators of the project and supervised all activities and aided in all aspects of the project, including development of the research questions and writing of the manuscript; C. S., B. M. and D. S. were the biostatisticians of the project and supervised the statistical methods used in the study, as well as reviewed all drafts of the manuscript; I. B. K., B. M. P., E. B. R. and X. S. obtained funding, collected the data, and reviewed and edited all drafts of the manuscript.

D. M. reports receiving research grants from GlaxoSmithKline, Sigma Tau, Pronova, and the National Institutes of Health for an investigator-initiated, not-for-profit clinical trial; travel reimbursement, honoraria or consulting fees related to conferences on diet and chronic diseases from the International Life Sciences Institute, Aramark, Unilever, SPRIM, Bunge, Foodminds, McKinsey Health Systems Institute, and Nutrition Impact; and royalties from UpToDate. B. M. P. is serving on the DSMB for a clinical trial of a device funded by the manufacturer (Zoll LifeCor) and on the Steering Committee for the Yale Open Data Access Project funded by Medtronic. There are no other conflicts of interest to report.

\section{References}

1. Harris WS, Kris-Etherton PM \& Harris KA (2008) Intakes of long-chain omega-3 fatty acid associated with reduced risk for death from coronary heart disease in healthy adults. Curr Atheroscler Rep 10, 503-509.
2. Harris WS, Poston WC \& Haddock CK (2007) Tissue $n-3$ and $n-6$ fatty acids and risk for coronary heart disease events. Atherosclerosis 193, 1-10.

3. Albert CM, Campos H, Stampfer MJ, et al. (2002) Blood levels of long-chain $n$ - 3 fatty acids and the risk of sudden death. $N$ Engl J Med 346, 1113-1118.

4. Albert CM, Hennekens CH, O'Donnell CJ, et al. (1998) Fish consumption and risk of sudden cardiac death. JAMA 279, 23-28.

5. Lemaitre RN, King IB, Mozaffarian D, et al. (2003) n-3 Polyunsaturated fatty acids, fatal ischemic heart disease, and nonfatal myocardial infarction in older adults: the Cardiovascular Health Study. Am J Clin Nutr 77, 319-325.

6. Burr ML, Fehily AM, Gilbert JF, et al. (1989) Effects of changes in fat, fish, and fibre intakes on death and myocardial reinfarction: diet and reinfarction trial (DART). Lancet iii, $757-761$.

7. Wang C, Harris WS, Chung M, et al. (2006) n-3 Fatty acids from fish or fish-oil supplements, but not alpha-linolenic acid, benefit cardiovascular disease outcomes in primaryand secondary-prevention studies: a systematic review. Am J Clin Nutr 84, 5-17.

8. Bushkin-Bedient S \& Carpenter DO (2010) Benefits versus risks associated with consumption of fish and other seafood. Rev Environ Health 25, 161-191.

9. Lenihan-Geels G, Bishop KS \& Ferguson LR (2013) Alternative sources of omega-3 fats: can we find a sustainable substitute for fish? Nutrients 5, 1301-1315.

10. Fried LP, Borhani NO, Enright P, et al. (1991) The Cardiovascular Health Study: design and rationale. Ann Epidemiol 1, 263-276.

11. Folch J, Lees M \& Sloane Stanley GH (1957) A simple method for the isolation and purification of total lipides from animal tissues. J Biol Chem 226, 497-509.

12. Lepage G \& Roy CC (1986) Direct transesterification of all classes of lipids in a one-step reaction. J Lipid Res $\mathbf{2 7}$, $114-120$.

13. Djousse L, Biggs ML, Lemaitre RN, et al. (2011) Plasma omega-3 fatty acids and incident diabetes in older adults. Am J Clin Nutr 94, 527-533.

14. Feskanich D, Rimm EB, Giovannucci EL, et al. (1993) Reproducibility and validity of food intake measurements from a semiquantitative food frequency questionnaire. $\mathrm{J} \mathrm{Am} \mathrm{Diet}$ Assoc 93, 790-796.

15. Lemaitre RN, Sitlani C, Song X, et al. (2012) Circulating and dietary $\alpha$-linolenic acid and incidence of congestive heart failure in older adults: the Cardiovascular Health Study. Am J Clin Nutr 96, 269-274.

16. Fretts AM, Mozaffarian D, Siscovick DS, et al. (2013) Associations of plasma phospholipid and dietary alpha linolenic acid with incident atrial fibrillation in older adults: the Cardiovascular Health Study. J Am Heart Assoc 2, e003814.

17. Ives DG, Fitzpatrick AL, Bild DE, et al. (1995) Surveillance and ascertainment of cardiovascular events. The Cardiovascular Health Study. Ann Epidemiol 5, 278-285.

18. Willet W (1998) Nutritional Epidemiology. New York: Oxford University Press.

19. Goyens PL, Spilker ME, Zock PL, et al. (2006) Conversion of alpha-linolenic acid in humans is influenced by the absolute amounts of alpha-linolenic acid and linoleic acid in the diet and not by their ratio. Am J Clin Nutr 84, 44-53.

20. Baylin A, Ruiz-Narvaez E, Kraft P, et al. (2007) Alphalinolenic acid, delta(6)-desaturase gene polymorphism, and the risk of nonfatal myocardial infarction. Am J Clin Nutr 85, 554-560. 
21. Mozaffarian D, Ascherio A, Hu FB, et al. (2005) Interplay between different polyunsaturated fatty acids and risk of coronary heart disease in men. Circulation 111, 157-164.

22. du Toit PJ, van Aswegen CH \& du Plessis DJ (1996) The effect of essential fatty acids on growth and urokinase-type plasminogen activator production in human prostate DU-145 cells. Prostaglandins Leukot Essent Fatty Acids 55, $173-177$.

23. Kamano K, Okuyama H, Konishi R, et al. (1989) Effects of a high-linoleate and a high-alpha-linolenate diet on spontaneous mammary tumourigenesis in mice. Anticancer Res 9, 1903-1908.

24. Fritsche KL \& Johnston PV (1990) Effect of dietary alphalinolenic acid on growth, metastasis, fatty acid profile and prostaglandin production of two murine mammary adenocarcinomas. J Nutr 120, 1601-1609.

25. Bourre JM (2004) Roles of unsaturated fatty acids (especially omega- 3 fatty acids) in the brain at various ages and during ageing. J Nutr Health Aging 8, 163-174.

26. Gerber $M$ (2012) Omega-3 fatty acids and cancers: a systematic update review of epidemiological studies. Br J Nutr 107, Suppl. 2, S228-S239.

27. Geleijnse JM, Giltay EJ \& Kromhout D (2012) Effects of $n-3$ fatty acids on cognitive decline: a randomized, doubleblind, placebo-controlled trial in stable myocardial infarction patients. Alzheimers Dement 8, 278-287.

28. Kim M, Nam JH, Oh DH, et al. (2010) Erythrocyte $\alpha$-linolenic acid is associated with the risk for mild dementia in Korean elderly. Nutr Res 30, 756-761.

29. Cherubini A, Andres-Lacueva C, Martin A, et al. (2007) Low plasma $N-3$ fatty acids and dementia in older persons: the InCHIANTI study. I Gerontol A Biol Sci Med Sci 62, $1120-1126$.

30. Simon JA, Fong J, Bernert JT Jr, et al. (1995) Serum fatty acids and the risk of stroke. Stroke 26, 778-782.

31. Park Y, Park S, Yi H, et al. (2009) Low level of $n-3$ polyunsaturated fatty acids in erythrocytes is a risk factor for both acute ischemic and hemorrhagic stroke in Koreans. Nutr Res 29, 825-830.

32. Khaw KT, Friesen MD, Riboli E, et al. (2012) Plasma phospholipid fatty acid concentration and incident coronary heart disease in men and women: the EPIC-Norfolk Prospective Study. PLoS Med 9, e1001255.

33. Wang L, Folsom AR \& Eckfeldt JH (2003) Plasma fatty acid composition and incidence of coronary heart disease in middle aged adults: the Atherosclerosis Risk in Communities (ARIC) Study. Nutr Metab Cardiovasc Dis 13, 256-266.

34. Wiberg B, Sundstrom J, Arnlov J, et al. (2006) Metabolic risk factors for stroke and transient ischemic attacks in middle- aged men - a community-based study with long-term follow-up. Stroke 37, 2898-2903.

35. Warensjo E, Sundstrom J, Vessby B, et al. (2008) Markers of dietary fat quality and fatty acid desaturation as predictors of total and cardiovascular mortality: a population-based prospective study. Am J Clin Nutr 88, 203-209.

36. Pan A, Chen M, Chowdhury R, et al. (2012) $\alpha$-Linolenic acid and risk of cardiovascular disease: a systematic review and meta-analysis. Am J Clin Nutr 96, 1262-1273.

37. Folsom AR \& Demissie Z (2004) Fish intake, marine omega-3 fatty acids, and mortality in a cohort of postmenopausal women. Am J Epidemiol 160, 1005-1010.

38. Oomen CM, Ocke MC, Feskens EJ, et al. (2001) Alphalinolenic acid intake is not beneficially associated with 10-y risk of coronary artery disease incidence: the Zutphen Elderly Study. Am J Clin Nutr 74, 457-463.

39. de Goede J, Verschuren WM, Boer JM, et al. (2011) Alphalinolenic acid intake and 10-year incidence of coronary heart disease and stroke in 20,000 middle-aged men and women in the Netherlands. PLOS ONE 6, e17967.

40. Pietinen P, Ascherio A, Korhonen P, et al. (1997) Intake of fatty acids and risk of coronary heart disease in a cohort of Finnish men. The Alpha-Tocopherol, Beta-Carotene Cancer Prevention Study. Am J Epidemiol 145, 876-887.

41. Ascherio A, Rimm EB, Giovannucci EL, et al. (1996) Dietary fat and risk of coronary heart disease in men: cohort follow up study in the United States. BMJ 313, 84-90.

42. Albert CM, Oh K, Whang W, et al. (2005) Dietary alphalinolenic acid intake and risk of sudden cardiac death and coronary heart disease. Circulation 112, 3232-3238.

43. Vedtofte MS, Jakobsen MU, Lauritzen L, et al. (2011) Dietary $\alpha$-linolenic acid, linoleic acid, and $n$-3 long-chain PUFA and risk of ischemic heart disease. Am J Clin Nutr 94 1097-1103.

44. Laaksonen DE, Nyyssonen K, Niskanen L, et al. (2005) Prediction of cardiovascular mortality in middle-aged men by dietary and serum linoleic and polyunsaturated fatty acids. Arch Intern Med 165, 193-199.

45. Brouwer IA, Katan MB \& Zock PL (2004) Dietary alphalinolenic acid is associated with reduced risk of fatal coronary heart disease, but increased prostate cancer risk: a meta-analysis. J Nutr 134, 919-922.

46. Larsson SC, Virtamo J \& Wolk A (2012) Dietary fats and dietary cholesterol and risk of stroke in women. Atherosclerosis 221, 282-286.

47. He K, Rimm EB, Merchant A, et al. (2002) Fish consumption and risk of stroke in men. JAMA 288, 3130-3136.

48. Hu FB, Stampfer MJ, Manson JAE, et al. (1999) Dietary intake of alpha-linolenic acid and risk of fatal ischemic heart disease among women. Am J Clin Nutr 69, 890-897. 